\title{
Historia Cultural de la Educación Especial en Chihuahua: Transformación de su cultura escolar 1970-2015
}

\author{
Cultural history of special education in Chihuahua: \\ Transformation of its scholar culture 1970-2015
}

\author{
Fernando Ponce Ramírez \\ Universidad Pedagógica Nacional del Estado de Chihuahua, Campus Delicias \\ fponce@upnech.edu.mx
}

\section{Resumen}

En el área de investigaciones relacionadas con la Historeografía de la educación, la mayoría de los estudios se ubican en las tendencias de narración simple de los hechos pasados, sobrevaloración de los documentos oficiales y destacamiento de personajes importantes. Frente esta prevalencia investigativa, una nueva perspectiva de hacer historia, rescata las representaciones culturuales de los sujetos comunes. Esta tendencia es llamada "Historia cultural", que estudia significados dentro de un espacio particular y el contacto de estos en el mundo social, donde son llenados de sentidos dados por representaciones prácticas y sociales del sujeto. En el caso de la Educación especial, los estudios historiográficos mayoritariamente se ubican también, en las tendencias tradicionales propias del historicismo, al carecer de un soporte sólido en representaciones, discursos compilados y documentados de lo sucedido en el periodo de tiempo que se pretende abordar. Sobresalen los estudios que se enmarcan desde los saberes modernos de la psicología, pedagogía, así como las que refieren al estudio de los modelos de integración e inclusión. Es desde esta última tesis que surge la necesidad de efectuar una investigación historiográfica que busca darle voz a los sujetos de la educación especial involucrados en la cotidianidad y las regularidades de la experiencia vivida día con día, describiendo las transformaciones se han producido en las prácticas educativas de educación especial en Chihuahua durante el periodo 1970-2015, desde una visión histórica cultural, mediante el análisis de entrevistas a profundidad, y la revisión de evidencias que se rescatan de ese periodo de tiempo.

\section{Palabras clave}

Inclusión, historia cultural, educación especial, cultura escolar.

\begin{abstract}
In the area of research related to the Historiography of education, most of the studies are located in the trends of simple narration of past events, overvaluation of official documents and highlighting important characters. Faced with this investigative prevalence, a new perspective of making history rescues cultural representations of common subjects. This tendency is called "cultural history", which studies meanings within a particular space and the interactions of these in the social world, where they are filled with meanings given by practical and social representations of the subject. In the case of special education, historiographical studies are mostly located, in the traditional
\end{abstract}


tendencies of historicism, lacking a solid support in representations, compiled and documented stories of what happened in the addressing time period. Studies that come from the modern knowledge of psychology, pedagogy, as well as those that refer to the study of integration and inclusion models, are amongst the rest, outstanding. It is from this last thesis that the need arises to carry out a historiographic investigation that seeks to give voice to the subjects of special education involved in daily life and the regularities of the experience of the day to day living, describing transformations that have taken place in the educational practices of special education in Chihuahua during the period 1970-2015, from a historical-cultural perspective, through an in-depth analysis of interviews, and the review of evidence rescued from that time period.

\section{Keywords}

Inclusion, cultural history, special education, school culture.

\section{Introducción}

El estudio cobra relevancia porque pretende rescatar las representaciones que sobre este periodo educativo sostienen los actores cotidianos de la educación especial. Cobra importancia así mismo, dado que busca identificar las constantes, pero también las coyunturas y rupturas que en este tiempo histórico se han producido, entretejiendo la vida de los docentes, padres de familia $y$ alumnos con discapacidad, con las políticas educativas enmarcadas en contextos sociales, e institucionales particulares.

La investigación documental de los hechos del pasado, implica una diversidad de procesos y formas para su construcción en una estructura sólida, entendible y digerible para el lector. La historiografía es conocida como el la producción escrita del conocimiento histórico y su consolidación como rama de las Ciencias Sociales se ha posicionado como punto de referencia entre las distintas disciplinas del saber (Fontana, 1982).

En el campo educativo, el objeto de estudio de la Historiografía de la Educación es la documentación, análisis e interpretación de la actividad de educar en algún espacio y tiempo del pasado. La historia recuperada del saber educativo es considerada con elementos sustanciales de una ciencia histórica, ciencia educativa y ciencia social. (Gulchet, 2006).
Para efectos de la presente investigación, dentro del campo disciplinar historiográfico-educativo, se toma a la Educación Especial como tema de estudio, disciplina relacionada con los conceptos de discapacidad, deficiencia, necesidades educativas especiales, diversidad, ceguera, sordomudez, inclusión entre otros.

El conocimiento de lo que circunda al tema de investigación, implica en la revisión de la literatura, rastrear los estudios históricos realizados para documentación, análisis e interpretación de los hechos y sucesos acontecidos diacrónicamente. En esa revisión se encuentran tres grandes tendencias que dividen el recorrido histórico de la Educación Especial en distintos momentos o épocas, definiciones , concepciones del sujeto. modelos de atención, implicaciones legales y jurídicas e institucionalización en los sistemas escolares.

La primera de ellas, denota una mirada global de la evolución y concreción en su quehacer para la atención al sujeto con discapacidad. Este análisis lo realiza a nivel macro, rescatando fechas importantes, personajes coyunturales, creación de instituciones, implementación de propuestas, así como evolución de modelos de atención, entrelazando todo esto a nivel mundial con una descripción inclinada a sucesos cronológicos. 
La segunda tendencia describe una análisis de la Educación Especial, establecida como sistema escolar. Su mirada la concreta en la interpretación del modelo educativo y su implementación en los centros educacionales. Se refieren caracterizaciones de los servicios, maestros, niños que están implícitos en atención a la discapacidad.

La última mirada marcada por los estudios históricos de Educación Especial, configura una análisis más específico de lo que sucede dentro de una institución, el impacto de un modelo y descripción de un personaje.

\section{Estudios históricos de educación especial- mirada internacional}

Las aportaciones de estos estudios muestran la característica de la narración cronológica en una línea del pasado al presente, destacando coyunturas, rupturas y transformaciones de los hechos implícitos en la deficiencia. Estos trabajos se desarrollan partiendo del análisis en los tiempos antiguos como pauta de su reflexión, y concluyen la reconstrucción del pasado con un punto de llegada a la época de integración e inclusión educativa en el siglo XX. El análisis principal es la atención al sujeto con una condición desfavorable física, mental o sensorial, desde las concepciones que existían en cada contexto. Estos documentos son situados en una descripción general de la antigüedad clásica o bien detalladas en culturas como Mesopotamia, Persia, Egipto, Grecia, Roma, entre otras. siguen un tránsito por la Edad Media, Edad Moderna, hasta llegar a las transformaciones educativas actuales (Vergara 2002, Castejón y Navas, 2002, Forteza 2005). Aguado(1995) en su obra monumental titulada La historia de las deficiencias, recorre el mismo trayecto del pasado remoto a la era actual, con datos muy específicos y detallados, proponiendo tres grandes revoluciones de la salud mental, que han sido marcadas por la especialización en la atención.
Dos estudios con enfoques de análisis multidisciplinar rescatan los cambios culturales en cada época respecto a la Educación Especial. Por una lado está el artículo Del padecimiento a la enfermedad: un camino hermenéutico (Jiménez, 2014), que brinda una interpretación de la concepción y significado en la intervención hacia la discapacidad. El otro estudio se titula del modelo del déficit, al modelo de Escuela Inclusiva (González, 2009), analizando exclusivamente el impacto de los modelos de atención a través del tiempo.

También se encuentran estudios que enlazan esta mirada generalizada de lo ocurrido en distintas épocas, lugares y culturas, a otra visión del desarrollo específico de la disciplina en un contexto determinado. Romero (2014), y Pérez (s/f) reconstruyen la aproximación de la Educación Especial desde este análisis global, y lo trasladan a la consolidación que existió en España, con la promulgación y trascendencia de distintas leyes, para la institucionalización de esta como atención educativa. Fernández (2011) toma como punto de partida la atención al deficiente sensorial en el siglo XVI, concluyendo su trabajo con el análisis de la concepción legal en ese país. Moreu (2009) posiciona otro estudio, en el análisis específico de tres países y sus interacciones respecto al tema: Francia, Alemania y España, son descritos desde sucesos ocurridos en el siglo XIX.

En esta misma tendencia se encuentra historiografía hecha en un contexto específico sin tomar como base los antecedentes ocurridos en otras partes del mundo. Un Estudio analiza lo ocurrido a partir del siglo XIX en Hungría (Kerl, 2009) mostrando un proceso de informalidad en la atención del sujeto, hasta propuestas médicas que se consolidaron como importantes. Estudios situados en el siglo XX sobre el tema en Brasil (Orru, 2006), Venezuela (Méndez, 2008), y Chile (2010), entrelazan distintos momentos 
que forjaron la evolución de Educación Especial hasta llegar a los servicios escolares.

Investigaciones que hacen referencia al proceso recorrido en España podemos encontrar estudios con datos desde el siglo XVI (Martínez, 2011) y XVII (García, 2009) que sitúan la propuestas e innovaciones pioneras en la época. Molina (2011) y González (2009, 2011) describen lo acontecido desde una inclinación legal, aportando elementos desencadenantes en la Constitución de 1812 y la Ley Moncayo en 1857, que forjarían la creación de instituciones, políticas y programas de atención. Casanova (2011), retoma el camino que marcó la Ley General de Educación en este país en 1970, narrando los cambios sociales que tuvieron impacto en una nueva concepción del sujeto deficitario. Estos últimos cuatro estudios terminan su análisis en la implementación legal del régimen educativo.

Otros estudios con una mirada específica abordan el seguimiento histórico de las declaraciones internacionales y su impacto en la concepción educativa del sujeto con discapacidad. López (2009) plantea un recorrido desde la Declaración Universal de los Derechos Humanos en 1948, hasta la Convención de los Derechos para las Personas con Discapacidad en el 2006. Este mismo análisis es propuesto en otro estudio específicamente para América Latina pero a partir de 1989 con la Convención de los Derechos del Niño, (Lauzurika, A., Dávila, P., Naya, L., 2009).

\section{Estudios históricos de educación especial, mirada en México}

Diversos estudios, han indagado el tránsito marcado por la educación especial en México, desarrollando descripciones que reconstruyen las huellas de las acciones más importantes respecto al surgimiento, consolidación y evolución en lo que respecta al tema. Las investigaciones identifican y reconocen la influencia de modelos, ideas, programas y leyes tomadas de otros lugares que transformaron la visión en la atención a la discapacidad.

Padilla (2009, 2010), reseña un panorama general de lo sucedido a finales del siglo XIX, distribuyendo hechos, bosquejos e impresiones de la percepción de los sujetos deficientes en ese tiempo. Recorre estas referencias de manera cronológica con un análisis detallado hasta las primera década del siglo XX. Osnaya (2004) en un estudio similar entrelaza cada acontecimiento ocurrido, trazando una perspectiva que va de lo general a lo particular en sucesos internacionales a partir del siglo XVI, hasta la creación de los servicios de atención en el sistema educativo en el siglo XX.

Un libro trascendental que traza el recorrido del país, se titula Memorias y Actualidad en la Educación Especial en México (SEP, 2010). Reconstruye desde la creación de la Escuela Nacional de Sordomudos en 1866 hasta el 2010, año del bicentenario de la independencia como nación. Sitúa detalles muy específicos, dividiéndolo en cuatro momentos de análisis, rescatando datos coyunturales que favorecen las transformaciones de lo que hoy se considera modelo educativo. Una reseña general de este libro es elaborada por Quiroz (2014) titulado El acontecer de la Educación Especial en México, que recorre los sucesos más importantes referidos y los enmarca como las coyunturas más significativas de lo que ha sucedido en el país.

Se encuentran estudios que analizan con una mirada más específica en los contextos estatales, englobando los eventos más importantes a nivel mundial, su traslado a la implementación de ideas y propuestas a México y su impacto en la entidades federativas. Encontramos el análisis de la educación especial en Sinaloa a partir del siglo $\mathrm{XVI}$, hasta el funcionamiento de los servicios 
en el régimen educativo de 1980, detallando personajes notorios y comunes, creación de instituciones $\mathrm{y}$ acciones particulares en ciertos municipios (Mota, 2010). Una investigación similar es propuesta para el estado de Durango, pero con una análisis en el periodo de 1958-1998 (Barraza, López, García, 1998). En el mismo tenor esta la descripción con referencia muy generales de lo ocurrido en el Distrito Federal, partiendo del análisis mundial en la atención al sujeto a partir de mediado del siglo XX. (Sánchez, 2004). El Departamento de Educación Especial en el Estado de Morelos, en el marco

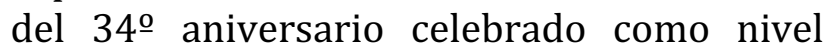
educativo redactó un boletín informativo con descripciones generalizadas de antecedentes en los servicios escolares, en las tres últimas décadas del Siglo XX.(SEP, 2013).

Dos estudios que analizan datos específicos en la dinámica impuesta por los servicios de educación especial a partir de la década de los 70s, describen situaciones impliciticas en ámbitos de atención con características particulares. Una de estas investigaciones analiza la adaptación del recurso de innovación Enciclomedia, para la accesibilidad de los alumnos con necesidades especiales. Refiere como se consolida la educación especial en el siglo XX, desde una mirada internacional con impacto en México en un trayecto que llega hasta el siglo XXI, con la utilización de esta tecnología en la aulas educativas (Soriano, García, Huesca, Rodríguez, 2006). Por otra partes está el estudio de indagación de la profesionalización del docente de este nivel educativo, recorriendo los distintos planes de estudio que han formado el perfil del maestro que está frente a esta atención. Hace consideraciones importantes de la evolución que ha tenido esta función, transformándose en una especialización que oferta licenciaturas y posgrados. (Guajardo, 2010).

\section{Estudios históricos de educación especial, miradas locales}

Otra de las perspectivas de realizar estudios históricos, centra el análisis de un periodo de tiempo específico mediante la interpretación de los sucesos en una institución, determinados por un modelo de atención o realizados por un personaje.

En este contexto, en España existen diversos estudios que recorren diversos puntos de análisis. Calderón (2009) centra su estudio en el boletín BILE de la Institución Libre de Enseñanza que pertenecía a la Institución de la Universidad de Madrid. Recopila e interpreta una documentación de noticias, comentarios y artículos en el periodo de 1878 a 1943 de lo que sucedía en España y en la mayor parte de Europa respecto a métodos y técnicas sobre la educación de sordomudos y ciegos. Un estudio similar de García (2009), recupera noticias del periódico "La gaceta regional" en Salamanca durante el periodo de 1974-1982, sobre alguna información que hiciera mención de la Educación Especial. Montes, S. Y Ramirez, F. (2009) también analiza artículos de la revista Escuela Moderna de España a principios del siglo $\mathrm{XX}$ en las aportaciones de temas referentes con la atención a la deficiencia de sordos, ciegos y débiles mentales.

Las instituciones son objeto de interés

por rescatar los significados en la consolidación de la educación especial. El Real Colegio de Sordomudos en Madrid pionera a lo largo del siglo XVIII Y XIX, es indagada por Gascón y Storch (2011); recobra los sucesos dentro de las actividades de la institución y su dinámica de atención a los sujetos a partir de 1795. Por otra parte, Martínez (2009) relaciona la influencia del oralismo en la educación del sordo, refiriendo las políticas, propuestas, implementaciones y desarrollo de la tendencia hacia esta metodología. En el análisis de la Escuela Central de Anormales en esta misma ciudad, se documenta el funcionamiento institucional durante las 
primeras décadas del siglo $\mathrm{XX}$, reconstruyendo las transformaciones que trascendieron dentro del centro y las relaciones con otras instituciones encargadas de atender a deficientes sensoriales $y$ mentales. Un estudio relacionado con dicha institución, lo propone Negrin (2009) rescata la importancia de la Sociedad Económica Matritense en su fundación, sostenimiento y promoción de políticas y programas para la Escuela de Sordomudos en los inicios del siglo XIX.

En México una institución que es precursora en la atención a la deficiencia sensorial es la Escuela Nacional de Sordomudos. Uno de los estudios de sitúa después de la mitad del XIX (Cruz, Cruz 2013), describiendo la atención a la persona sorda desde la atención médica rehabilitadora hasta una evolución a las técnicas educativas con el método oral. Vega (2013) analiza la historia de los certámenes para la aplicación de exámenes a sordos en el siglo XIX, valorando los avances educativos que tenían dentro de la atención escolar. Se refiere las ceremonias al obtener los resultados y la dinámica de los prefectos, aspirantes y profesores de la Escuela Normal. En otro estudio también de este último autor (Vega, 2013) realizado para la misma intuición refiere la dinámica de la escuela, respecto a los preceptos nacionales de la educación laica propuesta en las leyes de reforma del mitad del siglo XIX, y su necesaria modificación por la enseñanza de la fe que se trasmitía dentro de la institución.

Otra institución en México encontrada en el recorrido precursor de la educación especial, es la Escuela Normal de Especialización, descrita por Guemes (2009) documentando el contexto de la fundación promovida por el Instituto Médico Terapéutico en el DF. Se sitúa en las décadas de 1930 a 1950, refiriendo actividades, dinámicas vividas por los nuevos profesionistas en formación así como las tareas, actitudes y programas que promovían los profesores a los estudiantes, para la atención a la deficiencia. Guemes (2011) en otra propuesta analiza el proceso de institucionalización de la escuela a partir de 1927, siendo base fundamental para el establecimiento de la educación especial como una práctica educativa más científica para con los sujetos con discapacidad, formando en este oficio a maestros especialistas.

Dos estudios en España resaltan la participación de personajes en actividades importantes respecto al tema. La intervención de Sidonio Pintado Arroyo a principios del siglo XX en España respecto a la atención a sujetos con deficiencia en España. Los referentes son su vida, obra, participación y actividades que realizó. Otro estudio está implícito en un documento histórico de Juan Andrés y Morell escrito a finales del siglo XVIII cuyo contenido tiene que ver con reflexiones propias y referentes a otros personajes que han educado a personas sordomudas, recuperando desde un análisis la estructura de dicho escrito, interpretando su estilo y contenido. Moreno (2011) hace un estudio de Silvia Cruz Enciso fundadora del nivel de Educación Especial en el Estado de Hidalgo, desde su fundación en 1971 hasta la reorganización de los servicios que se dio con el momento de la integración educativa. Narra la labor que hizo un personaje durante casi treinta años en la consolidación institucional del nivel educativo.

También encontramos estudios que analizan el modelo de atención, destacando sus propuestas. Gutiérrez (2009) analiza la evolución del modelo médico en atención al déficit y el psicológico en la rehabilitación del mismo en México, refiriendo políticas, personajes, encuentros, instituciones, desde la creación de la primera escuela de sordomudos, teorizando el papel fundamental de las disciplinas en el establecimiento de la 
institucionalización de la educación especial como el establecimiento de clases especiales.

\section{Consideraciones ante los estudios históricos de educación especial encontrados}

En el análisis de este recorrido sobre las investigaciones históricas existentes sobre Educación especial, se perciben estructuras con tendencia cronológica a la construcción lineal de los sucesos. Se aborda la atención a la deficiencia con representaciones y discursos compilados que concentran el análisis de las instituciones, los documentos, individuos notorios y los modelos generalizados, es decir, investigaciones con tendencia centralista, siendo muy pocos estudios los que rescatan esencia de las particularidades en los sujetos y sus interrelaciones en la vida cultural.

De igual forma existe poca documentación sobre lo acontecido en el municipio de Chihuahua respecto lo sucedido en el tema de investigación. Por eso se propone como objeto de estudio el recuperar la historia cultural de los servicios en esta ciudad, tomando como punto de partida el año de 1970, que constituye una coyuntura trascendental con la formación del Departamento de Educación a nivel nacional.

Es desde esta última tesis que surge la necesidad de efectuar una investigación historiográfica que busca darle voz a los docentes involucrados en la cotidianidad y las regularidades de la experiencia vivida día con día, lo que da origen al problema : ¿Qué transformaciones se han producido en la cultura escolar de educación especial en Chihuahua durante el periodo 1970-2015, desde una visión histórica cultural?

\section{Objetivo General}

Interpretar y describir las transformaciones que se han producido en la cultura escolar de educación especial en Chihuahua durante el periodo 1970-2015, teniendo como enfoque una visión histórica cultural.
Objetivos específicos

- Se busca desarrollar la interpretación y descripción de la transformación de las cultura escolar en el periodo de tiempo referido, mediante los siguientes objetivos:

- Analizar el funcionamiento de las instituciones de educación especial en sus rupturas y regularidades en el periodo estudiado.

- Analizar y contrastar las diferencias en el quehacer didáctico de los profesores de educación especial, identificando el proceso de enseñanza - aprendizaje, realizado en el periodo de tiempo referido.

- Identificar las características de los contextos desarrollados en educación especial y la transformación de estos en el periodo estudiado.

\section{Técnicas e instrumentos}

En las técnicas e instrumentos se utilizan como herramientas básicas, la entrevista a profundidad de informantes clave, recuperación y análisis de evidencias (Fotografías, imágenes, contratos, materiales y recursos del trabajo profesional, entre otros).

La entrevista a profundidad, implica una revisión desde la literatura de los conceptos fundamentales, para cumplir con lo que se busca en la esencia del estudio. Su aplicación en este caso como técnica cualitativa se estructura a partir de objetivos concretos presentados. En el entendido de que resulta complicado determinar un número mínimo o máximo de entrevistados, pues no se busca un representación estadística, sino el análisis minucioso de la información que se obtenga de las conversaciones con los entrevistados.

El perfil de los informantes clave, será dirigido a docentes que trabajaron en educación especial en algún momento del 
periodo 1970 al 2015 por más de cinco años. Conforme se cubran los tópicos del guion realizado para la indagación de lo buscado en los objetivos, se verá qué tanta información hace falta $y$, a partir de ahí, determinar cuántas entrevistas más será necesario hacer.

El proceso de entrevista se divide en dos fases; la primera en el encuentro con el entrevistado, la recopilación de datos y el registro (Fernández 2003) La segunda, estudiará con detenimiento cada entrevista y se asignarán temas por categorías, para la interpretación de la información en futuro análisis.

González Martínez (2002) refiere que la adecuación de las herramientas y los procedimientos utilizados durante las fases del estudio así como la capacidad de corroborar las conclusiones con evidencias, son la base para validar y reconocer un buen trabajo (González Martínez en: Amezcua y Gálvez, 2002). Ante esto, se pretende solicitar las evidencias mencionadas para conformar una interpretación de la realidad con más sustento.

Los procedimientos de recogida de información que se van a usar en esta investigaciones son los siguientes: entrevistas a profundidad, grupos focales y evidencia históricas.

Por su parte, las fuentes personales que se consultan se especifican a continuación: Personal docente de educación especial/ Psicólogo de educación especial/ Trabajadora Social de educación especial/ Maestro de comunicación de educación especial.

Se aclara que en esta sección se hace la descripción detallada sobre la elaboración y aplicación de técnicas e instrumentos de recogida de información, sin embargo, los instrumentos completos son presentados en el apartado de anexos. Su participación implicaría un coste excesivo para las posibilidades de esta investigación. También hay que aclarar que el verdadero análisis de la información vendrá dado en el capítulo de resultados cuando se explore la información recogida de cada centro.

En la presente investigación se está en el momento del campo, terminandode abordar a los sujetos de la muestra, para iniciar el análisis de los resultados del instrumento. El proceso para el análisis de las estrevistas es el siguiente: Para el tratamiento de esta información se registraron las respuestas textuales más importantes de los participantes en fichas agrupadas por sus dimensiones e indicadores de la cultura escolar. Una vez reunida y clasificada esta información, se buscaran las proximidades y contrastes de respuestas entre los compañeros de una misma escuela por subdimensiones e indicadores para que, con esta diferenciación de resultados, se definiera el polo al que corresponde. Esta información fue complementada con las evidencias históricas obtenidas también para este estudio. La información obtenida fue organizada de la misma manera para cada uno de los participantes. El tratamiento de la información se llevará a cabo una vez grabadas las entrevistas. Se escuchó la grabación rescatando las respuestas más cercanas a las dimensiones que se anotaban textualmente en las fichas comentadas; se compararon las respuestas entre los profesionales de la misma escuela para seleccionar el contenido que recogiera la información más relevante.

\section{Conclusiones preliminares}

El proceso llevado hasta el momento ha propiciado conocimientos y posturas respecto a los elementos que rodean al objeto de estudio de la investigación, permitiendo crear un repertorio teórico para el análisis de los datos que el campo brinde.

A lo largo de estas páginas expondremos la trayectoria experimentada de la educación especial hasta llegar al concepto de inclusión, 
comenzando por referentes en los que educación especial se asociaba con el término de deficiencia, para posteriormente centrarnos en las implicaciones que tiene la cultura escolar.

El término alusivo a la educación especial, implica visualizar la atención a la discapacidad en los distintos momentos sociohistóricos, brindando el apoyo a las condiciones físicas, mentales o sensoriales que presentan una dificultad específica en la realización de acciones sociales en el contexto. Hasta finales del siglo XX estas condiciones son consideradas como deficiencias en el sujeto, es decir provocan la inadaptación a lo que la sociedad demanda. En la ciudad de Chihuahua atención a estas deficiencias, implicaba la desaparición y aislamiento por estar fuera de la regularidad en los estándares que cada contexto solicitaba. Se tiene muy arraigada la compasión que el ser humano despierta de manera colectiva, cambia la concepción de la asistencia que se comienza a brindar a las personas deficientes, con cuidados paliativos y de piedad en lugares específicos. Surge después una tendencia a la rehabilitación y cura de las capacidades afectadas para después visualizarlos gracias a las legislaciones como persona totalmente regulares.

Bayliss (1998), aborda la génesis de la palabra "especial" interpretada como una variación de la norma o bien asociada a condiciones médico-patológicas. Implica esta visión esa salida de lo ordinario, desde los ojos científicos en un paradigma médico. Cuando el contexto educativo se institucionaliza lo "especial" se constituye desde la pedagogía terapéutica que debía aplicarse fuera del ámbito de la educación ordinaria. Bayliss (1998), rescata que esta pedagogía instruye a los profesionales especialistas en ellas para definir que niño tenía problemas y qué intervención implementar para favorecer lo ordinario.
La creación de la Educación Especial, cumple con un derecho humanitario de atención de quienes habían estado abandonados, consolidando un avance en el desarrollo de los sistemas educativos, en tanto para otros contribuyó a institucionalizar la segregación (Allan, 2003; Barnes 1998; Oliver 1990; Vislie, 2003, 2006).

Ante esto es necesario saber que diversas personas han existido con limitaciones corporales en todos los tiempos, y son motivo de asombro, incomprensión, temor, diversión e ignorancia. En este marco diversas disciplinas han documentado el tema, algunas como la arqueología y paleopatología han analizado e interpretado las costumbres como el infanticidio y distintos remedios aplicados, en la existencia de anomalías congénitas, trastornos como gigantismo, enanismo y lesiones carenciales y degenerativas en culturas ancestrales muy remotas.

Las personas con deficiencia han sido conceptualizadas desde distintas visiones. Prevalecen dos de ellas en el periodo de la Antigüedad Clásica en culturas como Mesopotamia, Egipto, Grecia, Roma. La primera mirada se remonta con una tendencia demonológica, maléfica o mítica, aplicando una terapia de sortilegios, conjuros, magia, hechicería o bien el abandono y la aniquilación como solución. El segundo enfoque más científico las entenderá como patologías o enfermedades, analizada desde médicosfilósofos como Hipócrates, Galeno, Celso, provocadas por las culpas y voluntad de los dioses. (Vergara, 2002). Aguado (1995, p33) consolida estos enfoques como antecedentes de una primera revolución de la salud mental en el lapso de la prehistoria, hasta el primer Renacimiento a finales del siglo XVI, estableciéndose distintas concepciones y prácticas de acuerdo a cada región.

Con lo anterior se puede observar que el déficit se convierte en el concepto clave, implementándose dos formas de reacción en 
distintas regiones. Hasta antes de le Edad Media, se puede percibir la eugenesia y la marginación de las personas con deformaciones físicas y mentales (Palacios, 2008, pp37). En este modelo eugenésico se considera que la persona con discapacidad no merece vivir, pues su situación desgraciada no es digna. En un segundo modelo, impera la exclusión de las personas deficientes por miedo o compasión, ya no se comete la acción del infanticidio o la eliminación del sujeto, pero sigue una tendencia a morir por la omisión de cuidados.

\section{Referencias}

Aguado, A. (1995) La Historia de las Deficiencias. Valencia: Alfaplús.

Barraza, A. López, G. García, R. (1998). Cuarenta años de Educación Especial en Durango (1958-1998). Tesis.

Caiceo, J. (2010), Esbozo de la Educación Especial en Chile: 1850- 1980. Revista Educación y Pedagogía, vol. 22, núm. 57, mayo-agosto, 2010.

Calderón, M. (2009). Educación de los ciegos y sordomudos en el BILE. El largo camino hacia una educación inclusiva la educación especial y social del siglo XIX a nuestros días : XV Coloquio de Historia de la Educación, PamplonaIruñea, 29, 30 de junio y 1 de julio de 2009Vol. 1. 2009.

Castejon J.l. Navas L . Eds (2002) Unas bases psicológicas de la E. Especial. ECU Edit Club Universitario.

Cruz, J., Cruz, M. (2013).Integración Social del sordo en la Ciudad de México: enfoques médicos y pedagógicos (1867-1900). México DF. Revista Cuicuilco.

Fontana, F. (1982). Historia: análisis del pasado y proyecto social, Barcelona, Crítica, (Estudios y Ensayos), p. 9.

Forteza, D. (2005). Ayer y hoy de la educación especial en Pedagogía y educación ante el siglo XXI/ coord. por Julio Ruiz
Berrio, Gonzalo Vázquez Gómez, 2005, ISBN 84-608-0293-0, págs. 611-628.

Fernández, R. (2011) El camino hacia la integración Participación educativa, ISSN 1886-5097, №. 18, 2011 (Ejemplar dedicado a: Educación inclusiva: todos iguales, todos diferentes), págs. 79-90.

García, E. (2009). La Educación Especial en Salamanca a través de la prensa local: La gaceta Regional: 1974-1982. El largo camino hacia una educación inclusiva: la educación especial y social del siglo XIX a nuestros días : XV Coloquio de Historia de la Educación, Pamplona-Iruñea, 29, 30 de junio y 1 de julio de 2009 / coord. por María Reyes Berruezo Albéniz, Susana Conejero López, Vol. 1, 2009, págs. 59-72.

García, M. (2009). Iniciativas pedagógicas, motrices y sociales en el origen de la Educación Especial en España. El largo camino hacia una educación inclusiva: la educación especial y social del siglo XIX a nuestros días : XV Coloquio de Historia de la Educación, PamplonaIruñea, 29, 30 de junio y 1 de julio de 2009 / coord. por María Reyes Berruezo Albéniz, Susana Conejero López, Vol. 1, 2009, 235-248.

Gascón, A., Storch, J. (2011). El Real Colegio de Sordomudos en la primera mitad del siglo XIX. Antonio Gascón Ricao, José Gabriel Storch de Gracia y Asensio Participación educativa, ISSN 18865097, №. 18, 2011 (Ejemplar dedicado a: Educación inclusiva: todos iguales, todos diferentes), págs. 221-238.

González, E. (2009) Evolución de la Educación Especial: Del modelo del déficit al modelo de Escuela Inclusiva en El largo camino hacia una educación inclusiva: la educación especial y social del siglo XIX a nuestros días : XV Coloquio de Historia de la Educación, Pamplona- 
Iruñea, 29, 30 de junio y 1 de julio de 2009 / coord. por María Reyes Berruezo Albéniz, Susana Conejero López, Vol. 1, 2009, págs. 429-440.

González, T (2009). Itinerario de la Educación Especial en el sistema educativo: de la Ley Moyano a la Ley General de Educación. El largo camino hacia una educación inclusiva, Vol. 1, 2009-0101, ISBN 978-84-9769-244-1, pags. 249-260 01/2009; Source: OAI.

González, T. (2011). Modelos de escolarización: Trayectoria histórica de la Educación Especial. Educação e Filosofia Uberlândia, v. 25, n. 50, p. 691-716, jul./dez. 2011.

Guajardo, E. (2010) La desprofesionalización del docente de Educación Especial. Revista Latinoamericana de educación inclusiva volumen 4, numer 1-pp105129.

Guemes, R. (2009). La Identidad profesional de una generación de herederos legítimos en el campo de la Educación Especial. México CNIE.

Guemes, R. (2011). Una mirada en la historia. el proceso de institucionalización de la formación del docente en Educación Especial. CNIE XI.

Guichot, V. (2006) Historia de la Educación: reflexiones sobre su objeto, ubicación epistemológica, devenir histórico y tendencias actuales Revista Latinoamericana de Estudios Educativos (Colombia)pp9-10.

Gutiérrez, M. (2009). El modelo sicomédico: un legado de la medicina y la psicología para la Educación Especial. La construcción de un discurso hegemónico en 1921. El largo camino hacia una educación inclusiva: la educación especial y social del siglo XIX a nuestros días : XV Coloquio de Historia de la Educación, PamplonaIruñea, 29, 30 de junio y 1 de julio de 2009 / coord. por María Reyes
Berruezo Albéniz, Susana Conejero López, Vol. 1, 2009, págs. 73-84.

Hontañon, B. (2009). Nuevos enfoques y práctica pedagógica de la Educación Especial de la mano de Sidonio Pintado Arroyo. El largo camino hacia una educación inclusiva: la educación especial y social del siglo XIX a nuestros días : XV Coloquio de Historia de la Educación, Pamplona-Iruñea, 29, 30 de junio y 1 de julio de 2009 / coord. por María Reyes Berruezo Albéniz, Susana Conejero López, Vol. 1, 2009, págs. 261270.

Jiménez, G. (2014) Del padecimiento a la diversidad: un camino hermenéutico Revista Española de Discapacidad (REDIS), ISSN-e 2340-5104, Vol. 2, №. 2, 2014 (Ejemplar dedicado a: REVISTA ESPAÑOLA DE DISCAPACIDAD), págs. 185-206.

Kéri, K(2009). Los comienzos de la Educación Especial y la pedagogía curativa en Hungría. El largo camino hacia una educación inclusiva: la educación especial y social del siglo XIX a nuestros días : XV Coloquio de Historia de la Educación, Pamplona-Iruñea, 29, 30 de junio y 1 de julio de 2009 / coord. por María Reyes Berruezo Albéniz, Susana Conejero López, Vol. 1, 2009, págs. 271282.

Lauzurika, A., Dávila, P., Naya, L. (2009). El derecho a la educación de las personas con discapacidad. Una aproximación desde América Latina, en los últimos quince años. El largo camino hacia una educación inclusiva: la educación especial y social del siglo XIX a nuestros días : XV Coloquio de Historia de la Educación, Pamplona-Iruñea, 29, 30 de junio y 1 de julio de 2009 / coord. por María Reyes Berruezo Albéniz, Susana Conejero López, Vol. 1, 2009, págs. 147160. 
López, M. (2009). El derecho a la educación (inclusiva) de las personas con discapacidad en la Declaraciones Internacionales. El largo camino hacia una educación inclusiva: la educación especial y social del siglo XIX a nuestros días : XV Coloquio de Historia de la Educación, Pamplona-Iruñea, 29, 30 de junio y 1 de julio de 2009 / coord. por María Reyes Berruezo Albéniz, Susana Conejero López, Vol. 1, 2009, págs. 161170.

Martínez, P. (2009). Los inicios de la educación oralista en el Real Colegio de Sordomudos (1814-1823). El largo camino hacia una educación inclusiva: la educación especial y social del siglo XIX a nuestros días : XV Coloquio de Historia de la Educación, PamplonaIruñea, 29, 30 de junio y 1 de julio de 2009 / coord. por María Reyes Berruezo Albéniz, Susana Conejero López, Vol. 1, 2009, págs. 283-296.

Martínez, P. (2011). Hitos fundamentales de la educación especial en el siglo XIX. El Real Colegio de Sordo-Mudos. Participación educativa, ISSN 18865097, №. 18, 2011 (Ejemplar dedicado a: Educación inclusiva: todos iguales, todos diferentes).

Méndez, M. (2008). Conceptualización y Modelos de Atención de la Educación Especial en Venezuela. Conceptualización y Modelos de Atención,... Martha Méndez. AGORA Trujillo.Venezuela. AÑO 11- $\mathrm{N}^{\circ}$ 21ENERO - JUNIO - 2008.

Molina, R. (2009). La escuela central de anormales. El largo camino hacia una educación inclusiva: la educación especial y social del siglo XIX a nuestros días : XV Coloquio de Historia de la Educación, Pamplona-Iruñea, 29, 30 de junio y 1 de julio de 2009 / coord. por María Reyes Berruezo Albéniz, Susana
Conejero López, Vol. 1, 2009, págs. 297310.

Molina, M. (2011). La educación de los niños invidentes desde el siglo XIX hasta el inicio de su integración en los centros ordinarios. Participación educativa, ISSN 1886-5097, №. 18, 2011 (Ejemplar dedicado a: Educación inclusiva: todos iguales, todos diferentes), págs. 198-210.

Montes, S. Y Ramirez, F. (2009). Evolución de la Educación Especial a través de las revistas educativas. El largo camino hacia una educación inclusiva: la educación especial y social del siglo XIX a nuestros días : XV Coloquio de Historia de la Educación, PamplonaIruñea, 29, 30 de junio y 1 de julio de 2009 / coord. por María Reyes Berruezo Albéniz, Susana Conejero López, Vol. 1, 2009, págs. 85-92.

Moreno, A. (2011) Silvia Cruz Enciso, cofundadora de Educación Especial en el Estado de Hidalgo: Fundación, expansión y reorientación. (CNIE XI).

Moreu, A. (2009) La Pedagogía y la Medicina en los inicios de la Educación Especial ochocentista. Francia, Alemania y España en El largo camino hacia una educación inclusiva: la educación especial y social del siglo XIX a nuestros días : XV Coloquio de Historia de la Educación, Pamplona-Iruñea, 29, 30 de junio y 1 de julio de 2009 / coord. por María Reyes Berruezo Albéniz, Susana Conejero López, Vol. 1, 2009, 311-322.

Mota, R. (2010). La educación Especial en Sinaloa. Gobierno del Estado de Sinaloa.

Negrín, O. (2009). Labor pionera de la Real Sociedad Económica Matritense en la enseñanza de los sordomudos (18021808). El largo camino hacia una educación inclusiva: la educación especial y social del siglo XIX a nuestros 
días : XV Coloquio de Historia de la Educación, Pamplona-Iruñea, 29, 30 de junio y 1 de julio de 2009 / coord. por María Reyes Berruezo Albéniz, Susana Conejero López, Vol. 1, 2009.

Orru E. (2006). Educación Especial en Brasil: Una visión panorámica del proceso de inclusión de las personas con necesidades educativas especiales. Revista de Educación (Madrid).

Osnaya, A. (2004) La representaciones sociales de las unidades de servicio de apoyo a la educación regular. Tesis

Padilla, A. (2009). De excluidos e integrados: saberes e ideas en torno a la infancia anormal y la educación especial en México, 1920-1940. Frenia, Vol. 9, Fascículo 1, 2009, págs. 97-133.

Padilla, A. (2010). La educación especial en México a finales del siglo XIX y principios del XX: ideas bosquejos $\mathrm{y}$ experiencias. Revista Educación y Pedagogía, ISSN 0121-7593, Vol. 22, №. 57, 2010 (Ejemplar dedicado a: Historia de la educación de anormales y de la educación especial en Iberoamérica), págs. 15-30.

Pérez J. (s/f) Una aproximación histórica al concepto de Educación Especial.

Quiroz, M. (2014). El acontecer histórico de la Educación Especial en México. Revista electrónica de educación especial y familia 2014 número 1 (jun).

Romero C. (2014) Aproximación al campo de la Educación Especial: evolución y significado. Tesis.

Sánchez, P. (2004). La transformación de los servicios de educación especial en México. Conapred internet.

Soriano, C., García, J., Huesca, E., Rodriguez, S., (2006). Integración educativa en México y "Enciclomedia". Comunicación y pedagogía: Nuevas tecnologías y recursos didácticos, № 213, 2006, págs. 70-76.
SEP, (2010). Memorias y actualidad de la Educación Especial en México.

SEP MORELOS, (2013). Educación Especial en Morelos. Gaceta Boletín informativo.

Vega, M. (2013). La evaluación de los conocimientos en la Escuela de Sordomudos: Los certámenes públicos en el siglos XIX Mexicano. CNIE XII.

Vega, M. (2013). Avatares en la Introducción en la educación Laica en la Escuela Nacional de Sordomudos (1866-1875). CNIE XII.

Vergara, J. (2002) Marco Histórico de la educación Especial Revista: Estudios sobre Educación Volumen: № 2. Junio 2002 Pag: 129 - 143. Artículo DOI: Acceso libre.

Vergara, J. (2009). La primera historia de Educación de los Sordos en España: la carta del jesuita Juan Andrés y Morell (1740-1817) a su hermano Carlos sobre el arte de educar a los sordomudos. El largo camino hacia una educación inclusiva: la educación especial y social del siglo XIX a nuestros días : XV Coloquio de Historia de la Educación, Pamplona-Iruñea, 29, 30 de junio y 1 de julio de 2009 / coord. por María Reyes Berruezo Albéniz, Susana Conejero López, Vol. 1, 2009, págs. 101114. 
RECIE. Revista Electrónica Científica de Investigación Educativa Vol. 3, núm. 2, enero-diciembre 2017, pp. 855-867.

Ponce Ramírez, F. 